\title{
De Soccrent à Pluricapital : un modèle de développement régional inventé au Saguenay
}

\author{
Hubert Antoine Wallot ${ }^{1}$ \\ Université du Québec à Montréal
}

\section{Introduction}

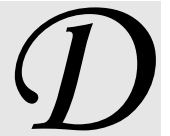

epuis maintenant plusieurs années, on considère la création de PME (petites et moyennes entreprises) comme un élément crucial du développement économique et de l'innovation ${ }^{2}$. À travers les efforts pour comprendre les phénomènes entourant la naissance, la survie et le développement de ces PME, l'attention s'est initialement portée sur le taux élevé de leur mortalité périnatale ou infantile : c'est-à-dire la grande proportion d'entreprises naissantes qui fail-

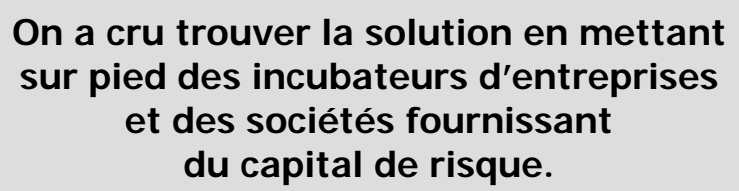

généralement localisés en milieu central, ces approches demeurent les plus utilisées.

Une autre tendance théorique et pratique a rapidement émergé selon laquelle le taux de naissance doit être accru par des actions en amont, à savoir avant la conception. En effet, la fécondation survient et de nouvelles entreprises naissent lorsque des entrepreneurs viennent en contact avec des opportunités. Le défi réside-t-il alors dans le manque d'entrepreneurs ou dans le manque d'occasions d'affaires? Selon la réponse qu'on donne, on trouve deux grandes lissent au cours des premières années de leur existence. On l'explique par leur difficulté à trouver suffisamment de financement ainsi que par leur manque d'expertise technique et gestionnelle, voire d'espace suffisant.

On a cru trouver la solution en mettant sur pied des incubateurs d'entreprises et des sociétés fournissant du capital de risque. Bon nombre des premières initiatives gouvernementales de soutien à la petite entreprise se sont situées à ce niveau. Les deux hypothèses implicites de ces solutions semblent les suivantes : 1 ) le taux spontané de naissance de PME est suffisamment élevé et 2) ce qui est à améliorer a trait au taux de survie à maturité.

Les approches rattachées à pareilles prémisses n’ont pas toujours apporté tous les bons effets souhaités, et les deux hypothèses sous-jacentes n'ont jamais pu être démontrées. Mais, dans la mesure où ces approches se prêtent à des applications autant en milieu régional qu'en milieu central urbain (quoique avec une efficacité différente) et que ces actions résultent de décisions d'instances provinciales ou nationales mises en œuvre par des fonctionnaires ou intervenants approches d'interventions dont s'inspirent notamment deux modèles québécois de développement économique régional par la création de PME : le CLD (Centre local de développement) et la formule Soccrent-Pluricapital, inventée au Saguenay.

\section{Les programmes gouvernementaux : soutenir l'entrepreneur}

Dans l'hypothèse qui postule une pénurie d'entrepreneurs motivés et aptes à démarrer une nouvelle entreprise, on repère certaines causes présumées et l'on cherche à agir sur elles : par exemple, des déficiences au niveau d'aspects psychologiques, familiaux et sociaux favorables à l'entrepreneuriat et au développement d'entrepreneurs. On escompte alors que le taux de naissance des PME croîtra par une action à long terme et une action à moyen terme sur l'éventuel entrepreneur. Dans l'action à long terme, qui est néanmoins logiquement la première, on cherche à éveiller le potentiel entrepreneurial latent dans certaines couches de la population, par exemple à travers la valorisation culturelle de l'entrepreneur. Dans l'action à moyen terme, qui prend pourtant la suite de l'action à 
long terme, il s'agit d'encourager la formation en gestion de l'individu au potentiel et à l'intérêt entrepreneuriaux développés, avec suivi et soutien de son projet d'entreprise qui lui tient généralement lieu de présentation. Ces actions supposent un processus assez long, non seulement en ce qui a trait aux changements dans la culture, mais aussi quant à la formation de l'entrepreneur qui requiert tout de même quelques années.

\section{II reste aux régions à assurer elles-mêmes leur développement à l'aide de leurs communautés d'affaires.}

Dans l'action à court terme, il s'agit de soutenir un entrepreneur qui a déjà un projet. Un grand nombre de programmes gouvernementaux cherchent à agir à ce niveau. Au Québec, le gouvernement a tenté de regrouper un ensemble de programmes à travers le concept de CLD. Cependant, le modèle d'intervention du CLD repose essentiellement sur le développement entrepreneurial des jeunes. Par ses différents programmes d'aide financière, il cherche à favoriser le développement de l'entrepreneurship, principalement auprès des jeunes de 18 à 35 ans. Le CLD se centre sur l'entrepreneur ${ }^{3}$, même si celui-ci se présente toujours flanqué d'un projet d'entreprise qui a son propre mérite: le CLD ne cherche pas à développer une entreprise, fut-elle à bon potentiel, dont le promoteur pourrait lui faire faux bond dans la poursuite du développement de son projet.

Les services offerts par le CLD consistent d'abord à encadrer le développement de l'entreprise par l'entrepreneur à divers niveaux et à diverses étapes : aide technique au développement du plan d'affaires, aide financière (subvention, prêt préférentiel) et aide au " réseautage ». Par la suite, le CLD suit l'entreprise démarrée avec son aide essentiellement pour fournir au promoteur un soutien dans la phase d'implantation et de surveiller son utilisation de l'aide financière. Les programmes gouvernementaux comme le CLD ont un certain coût économique puisque le CLD n'est pas une entreprise profitable et qu'il arrive parfois qu'il coûte aussi cher, sinon plus, en termes de fonctionnement, que les montants qu'il distribue. Il n’existe actuellement pas de données évaluatives concluantes sur le rendement économique de pareil type d'organisation.

\section{Prospection d'opportunités et circuits d'informations}

Dans une autre perspective, on cherche à améliorer le taux de naissance de PME par l'apport d'occasions (« opportunités ») d’affaires aux capacités fécondantes d'entrepreneurs. Normalement, ces occasions peuvent prendre des formes variées : innovations technologiques (produits nouveaux), innovations gestionnelles (par exemple les entreprises Cascades), innovations techniques (par exemple la mise au point de techniques de production plus efficaces et efficientes), innovation marketing (par exemple le hamburger McDonald). Mais l'occasion d'affaires peut prendre la forme d'une rencontre provoquée entre des partenaires potentiels qui s'ignorent comme tels ou encore entre des occasions d'affaires existantes et des entrepreneurs en recherche d'une occasion d'affaires. C'est ce qu'on peut appeler la fécondation in vitro, dont il sera question plus loin. En effet, la stimulation de la fécondité (c'est-à-dire de l'entrepreneurship) n'aura pas d'effet sur le taux de naissance de PME si les partenaires potentiels (les entrepreneurs et les occasions d'affaires) ne sont pas mis en présence. Cette opération suppose, comme élément majeur, une circulation de l'information que, dans la vision traditionnelle du marché libéral, on semble souvent prendre comme allant de soi. On peut vraisemblablement présumer que cela peut aller éventuellement de soi dans les grands centres urbains, lieux de confluence informationnelle et organisationnelle des grandes organisations nationales ou multinationales, ou encore plus spécifiquement dans les grandes entreprises, mais non dans les milieux périphériques. Or, ce sont ces derniers qui, depuis environ trois décennies, font l'expérience de la restructuration de leur économie au détriment du taux d'emploi sous la forme d'une modernisation des grandes entreprises régionales dans le secteur manufacturier de façon à ce qu'elles deviennent mondialement compétitives. La stabilisation de l'embauche dans ces secteurs n'a rien arrangé. De plus, dans certains régions, la présence de ces grands seigneurs (les grandes entreprises) offrant de très bons salaires à leurs employés, sans doute en compensation d'un accès généralement bon marché à des ressources naturelles, a découragé quelque peu la dynamique du développement des entrepreneurs, à la différence d'autres régions moins colonisées par la grande entreprise et plus créatives d'entrepreneurs, comme la Beauce. 
Il reste donc aux régions à assurer elles-mêmes leur développement à l'aide de leurs communautés d'affaires composées des grandes entreprises qui y opèrent et des autres gens d'affaires. De fait, la mise en commun de l'expertise technologique et administrative, des réseaux internationaux de communication, des connaissances des conditions locales rend possible l'introduction de nouvelles occasions d'affaires à partir d'informations, de technologies ou même d'équipements à l'extérieur à la région. Elle permet aussi de faciliter le démarrage de petites ainsi que de moyennes entreprises, notamment en instaurant un réseautage un peu sur le mode des grappes industrielles. Elle devrait avoir aussi un effet multiplicateur sur l'entrepreneuriat régional tout en constituant une solution préventive à un problème général dans les régions en Occident où les grandes entreprises sont invitées par la modernisation et la compétition à réduire substantiellement leur personnel.

\section{Naissance de Soccrent}

C’est dans ce contexte qu'au Saguenay, région plutôt nordique du Québec, on met sur pied, au tournant des années 1980, la Soccrent (Société en commandite de création d'entreprises). Il s'agit d'abord d'un contexte politique où de grandes sociétés multinationales avec des usines importantes dans cette région doivent, après diverses manœuvres de restructuration et de rationalisation ayant mené à des mises à pied, protéger leur image (même si ce n’est jamais présenté ainsi) en contribuant à la restauration d'un tissu économique régional. La Soccrent ainsi créée a donc comme première mission de créer de l'emploi dans la région à travers la création d'entreprises. Sa structure juridique est alors celle d'une société en commandite dont les actionnaires, les commanditaires, pouvant bénéficier d'avantages fiscaux relatifs au capital de risque, ont une responsabilité limitée à leurs engagements initiaux en capital, et rencontrent la condition qu'ils ne gèrent pas la société. Mais les commanditaires ont forcément un commandité qui va gérer les fonds. C'est Pluricapital, une entreprise privée à but lucratif à actionnaire unique, en l'occurrence un gestionnaire de type entrepreneurial, monsieur Adam Lapointe, qui est aussi professeur d'économie à l'Université du Québec à Chicoutimi. Pour réaliser sa mission, Soccrent s'est alors donnée, ce qui était une originalité à l'époque, trois fonctions : l'investissement, en région, de capital de risque, la création d'entreprises, l'incu- bation d'entreprises. Ces trois fonctions avaient pour objectifs principaux de promouvoir le développement régional et d'attirer en périphérie des activités économiques par le biais de la création d'entreprises manufacturières ou technologiques. La société ainsi créée se donne du coup divers indicateurs de performance qui vont par la suite lui donner raison en termes de création d'entreprises durables, d'emplois stables et de profits.

\section{Phase 1 : Un objectif de création d'emplois}

Pour des raisons politiques et aussi d'avantages fiscaux, les grandes entreprises régionales joignent Soccrent avec, comme première mission, la création d'emplois, même si le profit n'était pas d'emblée dédaigné, bien au contraire. La nouvelle société cherche à travailler sur des projets d'entreprises auxquels on peut déjà associer une expertise technique dans des personnes ou, maintenant le plus souvent, dans des partenariats avec d'autres entreprises. Cela est particulièrement intéressant lorsque ces partenaires sont étrangers et que les intérêts sont mutuels. Mais, avec le temps et l'expérience, la nouvelle société va agir de plus en plus comme promoteur plutôt que comme entrepreneur, car elle développe une expertise à monter des dossiers et à réunir les expertises nécessaires où l'on trouve déjà une expertise technique. C'est souvent selon le principe des grappes industrielles que la nouvelle société multiplie un certain nombre de projets d'entreprises autour d'une entreprise initiale qu'elle aura pu mettre sur pied. Elle ne sera plus gestionnaire une fois l'entreprise lancée. Elle identifie une idée à partir des ressources régionales, monte le dossier jusqu'à la décision d'implantation et trouve ensuite un gestionnaire. Donc, elle choisit de miser d'abord sur l'entreprise à créer et à laquelle adjoindre un gestionnaire plutôt que de miser sur un gestionnaire et avoir à vivre avec ses forces et ses faiblesses pendant longtemps. Son approche entrepreneuriale ressemble, par certains côtés, à l'intrapreneurship dans la grande entreprise. Ainsi, lorsqu'elle veut faire une construire une usine, une compagnie comme Alcan identifie un site, choisit une technologie et ce n'est qu'après la terminaison de la construction et au moment du démarrage du projet qu'elle embauche un directeur général. De plus, la société s’intéresse à des projets de type manufacturier et, avec le temps, d'une stature qui, notamment en raison des investissements 
importants, évoque la moyenne entreprise plutôt que la petite.

\section{Fécondation in vitro d'entreprises}

Le tandem Soccrent-Pluricapital est donc une entreprise originale agissant souvent comme une sorte d'agence "matrimoniale » où l'on retrouve tantôt la clinique de fertilité, tantôt les services de la mère porteuse : en quelque sorte, une organisation destinée à conjuguer des entrepreneurs à des occasions d'affaires selon un processus qu'on peut métaphoriquement dénommer fécondation in vitro ${ }^{4}$. Bien que cette idée d'une intervention d'un tiers dans une dynamique entrepreneuriale semble à contretemps de certaines perceptions scientifiques et populaires séculaires du processus entrepreneurial, certaines expériences en cours ou complétées questionnent les idées reçues.

La nouvelle société se distingue ainsi de l'approche gouvernementale classique, mentionnée plus haut, qui privilégie l'accent sur l'entrepreneur qui arrive quasi marié à un projet, son projet à lui. L'approche de Soccrent-Pluricapital comporte trois temps, si l'on peut dire : Pluricapital identifie une occasion d'affaires, trouve un partenaire technique et, ensuite seulement, embauche un directeur général identifié comme bon gestionnaire, un individu qui a des caractéristiques entrepreneuriales (et dans les faits, en très grande majorité, une formation universitaire en gestion) et qui va exploiter cette occasion d'affaires avec le partenariat technique. Pluricapital met cependant l'emphase sur l'entreprise à créer ou créée et non sur l'entrepreneur à soutenir. Si le gestionnaire de la nouvelle entreprise ne fait pas l'affaire, Pluricapital le change.

Pluricapital a une perspective particulière sur le développement économique régional. Cette entreprise considère qu'il est extrêmement difficile pour une petite entreprise de faire affaires avec une grande entreprise, pour diverses raisons telles que les normes de qualité, la capacité de production, les prix, etc. De plus, il s’y vivrait une situation de développement économique peu attrayante pour les entreprises de moyenne taille qui pourraient faire le pont (ou le relais) entre la grande entreprise et la petite entreprise. Elle croit que c'est ce fait de l'absence marquée de la moyenne entreprise qui pénalise l'intégration fonctionnelle et productive de la structure économique régionale. C'est pourquoi Pluricapital s'attaque à cette problématique en développant des opportunités d'affaires puis en les mariant avec les technologies appropriées, tout en respectant le développement des ressources existantes dans la région. Ce modèle de développement économique régional privilégie donc le développement d'entreprises manufacturières ou technologiques de taille moyenne de manière à permettre un relais fécond de développement entre les grandes et les petites entreprises.

Cette orientation, non fondamentale à l'approche, vient la compléter harmonieusement dans une perspective de développement régional solide. Donc, l'approche de Soccrent-Pluricapital vise le développement non pas par le haut (la grande entreprise) ou par le bas (le développement d'entrepreneur ou de l'entrepreneurship) mais, d'une certaine façon, par le mariage des deux à travers la mise sur pied d'une moyenne entreprise (investissement de cinq millions de dollars et plus), préférablement de type manufacturier. Ce modèle favorise donc le réseautage entre la petite et la grande entreprise.

\section{Pluricapital a une perspective particulière sur le développement économique régional.}

\section{Phase 2: Le réajustement de la mission}

La Soccrent est née avec une vocation de créer des emplois en région, dans une perspective de vocation d'entreprise de capital de risque où l'objectif premier n’était pas la profitabilité, même si elle était forcément au rendez-vous. Il s'agissait aussi, pour les grandes entreprises régionales, de se dédouaner moralement et politiquement des efforts qu'elles faisaient depuis quelques années en termes de modernisation et de rationalisation interne pour fin de compétitivité, efforts qui se traduisaient dans des coupures d'emplois dans la région. Les premiers succès de SoccrentPluricapital incitèrent les commanditaires à pratiquement doubler leur mise de fonds.

Puis, après ce dédouanage et les premiers succès de l'entreprise de capital de risque, notamment en matière de création d'emplois, il y eut une mutation importante. Les commanditaires, un ensemble de grandes entreprises enracinées dans la région, et qui avaient donné la mission initiale, revinrent implicitement et dans le concret quotidien à une mission 
plus traditionnelle. En effet, ils ralentirent ou cessèrent l'ajout de capital. Le développement s'en retrouva alors limité.

Aussi, de 1990 à 1996, la rentabilité devint un critère de décision aussi important que la création d'emplois. Cependant, les gestionnaires d'origine de Pluricapital, constatant que les grandes entreprises s'identifiaient plus facilement à des entités sectorielles, eurent l'idée de faire une approche sectorielle régionale dont le fonds SODEXFOR (1994) fut le prototype à partir de quoi fut développée et implantée l'idée des Para et des Soccrents.

\section{L'objectif opérationnel de Pluricapital est de mettre sur pied deux projets par année.}

\section{Phase 3 : Paras et mission de rentabilité}

La conjoncture amena donc Soccrent à mettre la rentabilité au premier plan de la mission. Par ailleurs, l'entreprise initialement appelée Soccrent se retrouva bientôt scindée en deux catégories d'entités juridiques distinctes : les Paras et les Soccrents, alors que Pluricapital demeurait toujours l'unique commandité.

Un Para est un regroupement d'entreprises solides et leaders dans un secteur donné, par exemple le bois. Les Paras correspondent donc à des secteurs d'activités au sein desquels le commandité Pluricapital développe des réseaux de contacts, lesquels vont permettre la révélation d'occasions d'affaires et, par suite, une structure de développement de projets principalement par grappes industrielles. Durant un développement de projet, des dérivés de produits - donc de nouvelles opportunités - peuvent émerger, à la façon des grappes industrielles. C'est à ce moment que la chaîne commence et que le choix des opportunités s'agrandit. L'effort se diversifie dans plusieurs secteurs d'activités, ce qui a déjà donné lieu à la constitution de six Paras différents : foresterie, métallurgie, agroalimentaire, technologie, chimie, énergie. Ce sont les Paras qui financent le développement de projets, non leur implantation. La composition des Paras peut varier dans le temps.

Par ailleurs, comme l'était le Soccrent initial et désormais dissous, un Soccrent est une entreprise de capital de risque formée en vue de la création d'entreprises. En général, le Soccrent se spécifie par un domaine d'intérêt semblable à celui d'un Para, et les commanditaires sont des organisations qu'on retrouve souvent autant dans le Para que dans le Soccrent du même domaine. Le Soccrent est donc l'organisation qui peut investir dans l'implantation d'un projet préparé à la commande d'un Para.

Au fond, l'arrimage des Para et des nouveaux Soccrents, qui ne se recoupent pourtant jamais complètement, repose sur l'élaboration progressive de réseaux stratégiques administrés par une équipe multidisciplinaire, celle de Pluricapital, dont un des objectifs principaux est de provoquer des investissements créateurs d'entreprises dans les secteurs à forte croissance, manufacturiers ou recourant à des technologies de pointe.

Enfin, Pluricapital est, d'une certaine façon, une entreprise de services visant à développer les projets, un peu à la manière d'un R\&D intrapreneurial qu'utilisent les Paras qui financent le développement des projets. Dès qu'un projet d'entreprise est développé jusqu'à l'étape de préimplantation, il est offert pour vente, en priorité à des organismes du Para concerné ou au Soccrent apparenté. Il y a donc là, après fécondation in vitro, transfert d'embryon. Il est entendu que la vente d'un projet doit couvrir, pour fins de remboursement, les frais de développement du projet par Pluricapital qui, étant une entreprise à but lucratif, doit y trouver aussi un profit. Par ailleurs, Pluricapital est aussi, dans les faits, la société qu'on sollicite pour gérer les Soccrents.

\section{Rentabilité des Soccrents et de Pluricapital}

Malgré un objectif commercial explicite (le profit), tant pour les commanditaires à travers les Soccrents qu'au niveau de la société gestionnaire commanditée, Pluricapital, cette dernière, à travers son actionnaire unique et président-directeur général, conserve comme valeurs majeures, sinon comme mission, le développement régional que toutefois elle identifie non pas à des individus, mais à des entreprises issues d'occasions d'affaires, et principalement dans les secteurs vitaux, le secteur manufacturier et le secteur technologique. L'objectif opérationnel de Pluricapital est de mettre sur pied deux projets par année. Les projets proviennent d'une opportunité d'affaires soit techno- 
logique, soit manufacturière, soit de richesse régionale. Le rôle de Pluricapital est donc de développer les différents projets, de trouver le financement nécessaire au démarrage et de faire fructifier le capital de risque des sociétaires. En outre, Pluricapital conseille le gestionnaire ou l'entrepreneur et contribue à maximiser sa rencontre avec des opportunités d'affaires. Pluricapital consolide également les réseaux, fait croître l'économie régionale et stimule l'internationalisation des activités, des entreprises et des projets.

À la fin de la décennie 1990, Pluricapital avait un bilan impressionnant. Active depuis 1986, Pluricapital regroupait 35 sociétaires et supervisait une trentaine de placements d'affaires. Pluricapital avait généré plus de 2000 nouveaux emplois en 12 ans et gérait des actifs de réseau de $350 \mathrm{M} \$$, pour un capital sous gestion de $32 \mathrm{M} \$$. Pluricapital avait acquis une réputation enviable et une crédibilité solide auprès de ses partenaires privés et publics. Pour les années 19931998, Pluricapital a obtenu pour ses commanditaires un rendement nominal annuel de 42,04 \% grâce à son réseau stratégique, un partenariat privé, son approche proactive et son équipe professionnelle. Depuis, compte tenu de la conjoncture économique, elle a obtenu pour ses commandités un niveau moyen supérieur à ce qu'on rencontre dans ce genre d'investissement pour la période, soit environ $11 \%$.

Néanmoins, précédemment très dédié à l'innovation et au développement de projets, à trouver les opportunités et les entreprises partenaires éventuelles ainsi qu'aux phases de préincubation et d'incubation, Pluricapital connaît deux phénomènes nouveaux. Lorsque, pour un projet, il y a peu d'expertise, notamment technique dans la région, Pluricapital tend à moins faire refaire à un entrepreneur local, pour l'introduire au projet monté, la démarche déjà faite par l'entreprise pour le développer, car cela alourdit le processus en introduisant des délais et des incertitudes. Donc, Pluricapital devient alors l'entrepreneur à la manière des banques d'affaires privées américaines. En second lieu, une condition fondamentale de succès des projets est l'apport suffisant et la continuité des capitaux pour procéder, depuis l'analyse jusqu'au prédémarrage de projet. Or actuellement, le marché du capital de risque, profit oblige, se déplace vers le développement de projets ayant déjà franchi l'étape plus risquée du prédémarrage, et ceci n'est pas sans influencer la structure Soccrent-Para-Pluricapital;
Pluricapital doit alors en tenir compte et s'adapter pour survivre.

\section{Phase 4 : L'extra régional et l'international}

Pendant tout ce temps-là, pendant que les grandes entreprises fournissent des fonds, l'expertise du développement et de l'implantation de projets s'est développée dans Pluricapital, dans le commandité. Son équipe a ainsi acquis une expérience régionale et sectorielle dans le démarrage d'entreprises. Jusqu'au moment où le marché régional du capital de risque s'est saturé parce qu'entre-temps sont arrivées d'autres sociétés de capital de risque à vocation régionale, comme la FIDQ (Fonds régional d'investissement Desjardins) qui empiétait directement sur la mission des divers tandems de Soccrent avec Pluricapital, laquelle firme devenait moins compétitive en raison des avantages fiscaux de ces nouveaux fonds publics. Ensuite, Pluricapital avait un fond qui était rendu à maturité. Et le coût des nouveaux fonds sur le marché était moins élevé que celui de Pluricapital et offraient beaucoup plus de disponibilité.

Par ailleurs, la société Pluricapital, le commandité de tous les Soccrents, a toujours été une entreprise privée, et donc la rentabilité était un de ses objectifs. Elle avait deux choix : ou prendre de l'expansion dans un nouveau marché, ou trouver une nouvelle vocation dans le secteur privé à travers le développement de l'expertise acquise... Comme par hasard, quand le président de Pluricapital se rendit à Montréal pour rencontrer les dirigeants de la Caisse de Dépôt, en 1998, ces derniers, constatant l'unicité de l'expérience de l'entreprise saguenéenne, lui proposèrent de mettre un bon capital à sa disposition à condition de ne pas borner le lieu d'intervention au Saguenay, mais de plutôt investir là où Caisse intervient. Alors, Pluricapital apparaît non plus simplement comme un fonds de capital de risque, mais d'abord comme une entreprise experte dans la découverte ou le montage d'opportunités, dans la préparation d'évaluation opérationnelle de projets soumis, de devis financiers ou de propositions d'affaires, puis de suivi intensif de l'entreprise mise sur pied. Le partenariat de la Caisse de Dépôt ouvre tout le marché. Mais s'il y eut l'initiation de trois projets, dont deux au Québec et un en Pennsylvanie, la Caisse de Dépôt se réorienta vers la fin de 2001 et se retira du secteur du démarrage d'en- 
treprises. Actuellement, les fonds sous gestion par le commandité Pluricapital sont autour de 35 millions \$.

Par ailleurs, dorénavant, l'expertise de Pluricapital, jadis strictement régionale, est offerte à tous. Cette expertise, les autres fonds de capitaux spécialisés ne l'ont pas, sauf dans les grandes villes. Pluricapital, au lieu d'être un fonds spécialisé, prend plutôt toute l'activité du placement du début à la fin (y compris le désinvestissement). Ce qui facilite les choses, c'est la présence d'équipes multidisciplinaires, de gens avec un esprit entrepreneurial et de gens avec des expériences différentes. Car, en dernier ressort, Pluricapital demeure l'entreprise d'un entrepreneur, et c'est ce qui fait sans doute que sa multiplication, et surtout la multiplication de son succès, ne vont pas nécessairement de soi.

En raison de l'esprit entrepreneurial qui y préside, beaucoup de gestionnaires de Pluricapital sont devenus des gestionnaires d'entreprises créées ou acquises par Pluricapital. Une personne œuvre dans Pluricapital depuis le début, il y aura bientôt 20 ans, soit son président fondateur et unique actionnaire, monsieur Adam Lapointe.

\section{Conclusion}

Pluricapital n'est pas simplement un concept gestionnel, mais un phénomène social qui révèle la dynamique tant de l'entrepreneur que de la grande entreprise en milieu régional. Pluricapital demeure une entreprise entrepreneuriale, et cette caractéristique contribue à maintenir, malgré son évolution, sa contribution régionale. Comme entreprise profitable, elle demeure sujette aux contrecoups du marché et peut-être même de la trajectoire de ses gestionnaires. La vocation de l'entreprise a changé, mais le fait que Pluricapital soit une entreprise de petite taille en termes d'employés et qu'elle n'ait qu'un seul actionnaire qui demeure au Saguenay, cela comporte des conséquences notables pour la localisation des ressources. Il en serait évidemment autrement pour une grande entreprise. Le modèle Soccrent-Pluricapital est un modèle séduisant. Comment se fait-il qu'on ne l'ait pas reproduit ? Nous y voyons diverses raisons. La première tient à l'approche qui veut agir en amont en provoquant la rencontre d'occasions d'affaires avec des gestionnaires. C'est une approche contraire aux perspectives courantes. La seconde, c'est une approche conçue pour la création d'entreprise en milieu périphérique, alors que les organismes susceptibles de s'intéresser à la création d'entreprises sont en milieu centraux et pensent que leurs modèles d'intervention, valables dans ce type de milieu, valent aussi pour la périphérie. La dernière raison a trait plus spécifiquement à la fonction du commandité Pluricapital, car il y a, pour que cela puisse émerger, la condition d'un gestionnaire exceptionnellement habile et habité par un esprit à la fois régionaliste et entrepreneurial qui croit en ce modèle.

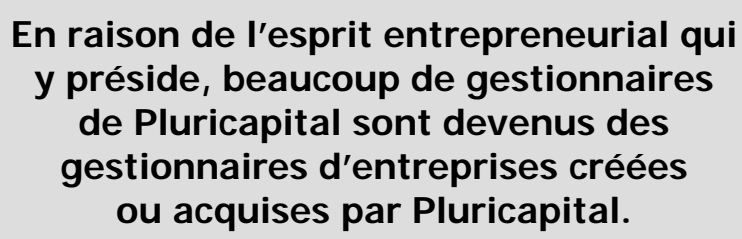

\section{Notes et références}

1 Hubert Wallot est professeur titulaire de gestion et responsable du programme court en entrepreneuriat à la Téluq (UQAM). Il est détenteur d"un diplôme en management de l'Université McGill, d'un MBA et d'un Ph.D. en management de l'Universié Laval, d'une maîtrise de Harvard et d'une scolarité de doctorat de l'Université de Paris. Il est aussi psychiatre et, à ce titre, professeur de clinique à l'Université Laval. Il s’intéresse aux aspects psychologiques de l'entrepreneuriat et à l'organisation des services de santé.

2 D’Amboise G. (1997). Quelle gestion stratégique pour la $P M E$ ? Presses Inter Universitaires, $2^{\mathrm{e}}$ Édition, p. 14-15.

3 Wallot H. et P. Barbeau (2001). « Entre l'entrepreneur et l'entreprise, faut-il choisir ? », Organisations et territoires, vol. $10, \mathrm{n}^{\circ} 2$, printemps-été, p. 61-80.

4 Wallot H. et S. Dubé (1995). « Magnitube. In Vitro fertilization of a Business », Gestion 2000, tome 1, p. 75-89; Wallot H. et S. Dubé (1998) « Incubation, capital de risque et création in vitro d'entreprise ", Revue organisation, vol. 7, $\mathrm{n}^{\circ} 1$, p. 57-69. 


\section{Publicité}

\section{Revue Organisations et territoires}

Site Web 